\title{
Oral Squamous Cell Carcinoma and the Cutting Edge of Nanotechnology
}

\author{
Rituraj Bharadwaj ${ }^{1}$, Subhash Medhi ${ }^{1, *}$ \\ ${ }^{1}$ Laboratory of Molecular Virology and Oncology, Department of Bioengineering \\ and Technology, Gauhati University, Guwahati, India \\ *Corresponding author: Subhash Medhi, Laboratory of Molecular Virology and \\ Oncology, Department of Bioengineering and Technology, Gopinath Bordoloi \\ Nagar, Jalukbari, Gauhati University, Guwahati, Assam 781014, India. Tel: \\ +919954485246, +917002485869; E-mail: subhashmedhi@gauhati.ac.in
}

DOI: $10.30699 / \mathrm{mci} .4 .2 .36$

Submitted:26 January 2020

Revised: 19 February 2020

Accepted: 16 March 2020

e-Published: 1 April 2020

Keywords:

Carcinoma, Squamous Cell

Tobacco Smoking

Smokeless Tobacco

Betel Quid

Alcohols

Nanotechnology
Cancer is a group of diseases characterized by uncontrolled cellular growth or proliferation. Oral squamous cell carcinoma (OSCC) is a group of cancers mainly affecting the buccal cavity and associated area, with high rate of mortality and morbidity. Chronic consumption of alcohol, both smoking and smokeless tobacco, and betel quid are considered as the prominent determinants for the initiation and development of OSCC. The current treatment strategy of OSCC is associated with the common side effects of non-specific anticancer drug delivery. In addition, the treatment also deals with the recurrence of OSCC after completion of the therapy, which is responsible for its high mortality rate. Nanotechnology is an emerging discipline in drug delivery, disease diagnosis, and imaging. Nanoparticles are a group of colloidal carriers with approximately 1-1000 $\mathrm{nm}$ size range. Nanoparticles, due to large surface area to volume ratio, display different physicochemical characteristics favorable for their use in chemotherapy. The nanoparticles with options for modifications in their sizes, surface charges, drug payload, and drug bioavailability can be used as a drug delivery system. The dynamic and flexible nature of nanoparticles can be employed to both active and passive targeting of cancer cells combating the usual side effects as well as other complexities associated with the conventional chemotherapy.

The nanoparticles can be regarded as the future of drug delivery in cancer therapy.

(C) 2020. Multidisciplinary Cancer Investigation

\section{INTRODUCTION}

Cancers can be defined as a group of diseases mostly characterized by uncontrolled and nondifferentiated growth of cells. They mostly occur due to the acquisition of somatic alteration in the genome of the cells, resulting in alteration of the function of cancer gene(s) [1]. It actually highlights an imbalance in the multi-cellular coordination in an organism, which cheats in a way affecting the level of fitness $[2,3]$. Cancer possesses transformed neoplastic cells gathering somatic molecular alteration and there always remains a continuous interplay among the cancerous and noncancerous cells including the cells of inflammatory and immune response. Thus, the innate and adaptive immunity of an organism is reported to influence the evolution of cancer [4]. Non-communicable diseases are recognized for the majority of death occurrence globally, and cancer is on the top of this chart leading to decrease in life expectancy in almost every nation of the globe [5]. Detection of around 18.1 million of new cancer cases and 9.6 million of deaths occurrence are reported for the year 2018 throughout the world; moreover, it is estimated that the risk of getting cancer before reaching the age of 
75 years is approximately $20 \%$ out of which $10 \%$ death is estimated [5].

\section{Oral Cancer and Oral Squamous Cell Carcinoma}

Oral cancer relates to those malignancies mainly affecting oral cavity, associated area of the pharynx, lips, buccal mucosa, gums, tonsils, uvula, palate, and vestibules. Oral cancer is most commonly expressed as oral squamous cell carcinoma (OSCC), since $90 \%$ or more of it histologically originates in the squamous cells [6]. Among different parts of the oral cavity, the pharynx is the most common site, followed by tongue and other parts for the development of OSCC [7]. OSCC is more prevalent in males than females. It has a high level of differentiation and strong tendency toward lymph node metastasis [8]. The disease stage at diagnosis is the most important parameter to determine the risk of dying from OSCC [9]. Early detection of OSCC at stage 1 results in a five-year survival rate of $80 \%$ as compared to its detection at stage 4 , which is $20 \%$ [10]. In advanced OSCC cases, the metastasis usually starts at the regional lymph nodes of the neck before getting spread out to other secondary sites, and thus it is considered that early diagnosis of the lymph nodes involvement is crucial for efficient therapeutic outcome [11].

\section{Etiology and Epidemiology of Oral Squamous Cell Carcinoma}

The etiology of OSCC seems to be multifactorial including genetic predisposition, as well as environmental and behavioral factors [12]. Lifestyle factors such as smoking tobacco, alcohol consumption, and betel quid chewing prominently contribute to the development of OSCC [1315]. Apart from the aforementioned risk factors associated with lifestyle, infection with human papilloma virus (HPV) also contributes to the development of OSCC [16]. Although, tobacco smoking and alcohol consumption still contribute to $75 \%$ of overall OSCC cases [17], it is even reported that chronic abuse of both of them has 38-48 odds to develop OSCC as compared to non-drinkers and nonsmokers [18]. Alcohol is casually associated with the occurrence of OSCC, as reported, consumption of alcohol (30 $\mathrm{g}$ of ethanol/day) in males and (15 $\mathrm{g}$ of ethanol/day) females may lead to the development of cancer [19]. Acetaldehyde, the chief metabolite of ethanol, is classified as Group-1 carcinogen by
IARC, implying a close association between alcohol consumption and OSCC occurrence [15]. Betel quid is a lifestyle-related carcinogenic agent used by the Asian population throughout the globe and stands in the fourth place after tobacco, alcohol, and caffeine beverages in terms of psychoactive habits [20]. Approximately, 600 million betel quid consumers are reported throughout the world, $10 \%$ of them are from South-East Asia and South-Pacific regions [21]. Nitrosamine and alkaloids found in betel quid are potent carcinogens and have the capability of developing pre-cancerous lesions in the oral cavity, which further get transformed into OSCC [14]. Smoking cigarettes or any other forms of tobacco have much deleterious effects on human health. The smoke mostly consists of harmful chemical compounds such as tobacco-specific nitrosamines (TSNAs). TSNAs, like N-Nitrosonornicotine (NNN) and nicotine-derived nitrosamine ketone (NNK), are well known carcinogens [22]. The most prominent carcinogen presents in the tobacco smoke is benzo[a]pyrene and it is claimed that it can induce OSCC in most of the smokers [13].

OSCC stands at the 6th place among all types of cancer in the world. It is more common in the developing nations as compared to the developed ones and it is reported that the incidence rate of OSCC in males vary from 1 to 10 cases per 100,000 inhabitants of many nations. OSCC even ranks among top three of all commonly occurring cancers in South-Central Asia [23]. A sharp increase in the incidence of OSCC is observed in Denmark, France, Germany, Scotland, and the Eastern Europe, and to a lesser extent in Australia, Japan, USA, and New Zealand [23]. South-and South-East Asia are considered to have high incidence of OSCC along with Eastern and Western Europe, parts of Latin America, and Caribbean and Pacific regions also do contribute to OSCC in the global scenario [24]. Approximately, 67,000 new cases were detected and registered in the European Union (EU) in 2004. Oral and pharyngeal cancer stands the 7th place in the EU [25]. But in case of mortality, the highest rate belongs to Eastern Europe [24]. The USA reported about 35,000 new OSCC cases in 2004 and the patient load is currently tripled [24].

\section{Current Treatment of Oral Squamous Cell Car- cinoma}

OSCC has a prominent impact on public health 
worldwide as oral cavity plays an important role in human necessary life functions including breathing, eating, and speaking. OSCC and the malignancy caused by it lead to death in most of the cases. The contemporary treatment of OSCC involved chemotherapy, surgical resection, radiotherapy, and transplant immunotherapy of hematopoietic stem cells alone or in combination [26]. Even surgery combined radiotherapy and chemotherapy may increase the life expectancy in patients with advanced OSCC. Numerous anti-neoplastic drugs are employed thus far for the conventional chemotherapy in OSCC cases. Over a century, surgery is a recognized and well-accepted treatment for the majority of the OSCC cases. But in most of the advanced OSCC cases, radiotherapy is always employed along with the surgery [27]. Generally, when functional or aesthetic loss of structures occurs in the oral cavity, reconstructive surgery is always followed by resection in OSCC cases. This includes loss of part of the tongue, buccal mucosa, and a portion of mandible and floor of the mouth [27]. The pharynx is one of the common sites for OSCC occurrence, which starts from the superior surface of the vault to nasopharynx up to the border of pharyngoesophageal junction. Neoplasm of nasopharynx is mostly treated with surgery and in combination with chemotherapy [27]. But in case of recurrence or malignancy in nasopharyngeal carcinoma, surgery is rarely employed. If the involved site is oropharynx, combined chemotherapy and radiation therapy is the preferred choice. Surgery is feasible in case of recurrent OCSS or the carcinoma of salivary gland origin [27]. In case of hypopharynx, if the cancer is detected at the early stages ( $\mathrm{T} 1$ and $\mathrm{T} 2$ ), it can be treated by transoral laser microsurgery or radiation therapy combined with chemotherapy. And in case of the detection of supraglottic larynx carcinoma at early stage, it is treated with radiation or surgery [27]. Combined therapy to enhance the effect of therapeutic regimen including radiation has a long history. Successive trials initially made in 1960s and then in 1970s hypothesized that the chemotherapy inhibiting DNA repair mechanism would contribute to the synergistic effects of radiation therapy in cancer treatment [28]. Although the radiation increases the efficacy of the chemotherapy to treat OSCC, lack of specificity is its major drawback [28]. Thus, improved radiosensitizers may help to selectively kill the tumor cells leaving behind the normal cells [28]. Radiotherapy is also employed to treat OSCC apart from radiation therapy, chemotherapy, and surgery. The external beam radiotherapy (EBRT) and/or brachytherapy are employed in concurrent with chemotherapy for late stage treatment of OSCC [29]. EBRT is used in three situations: as an adjuvant to primary surgery to enhance the specific control at the site, as primary treatment in case the patient cannot tolerate surgery, and as salvage treatment in recurrent OSCC cases [29]. Modifying the EBRT fractionation to treat OSCC allowed intensifying the dose of radiation either by increasing the total dose with hyper-fractionation or shortening the fraction using the accelerated fraction radiotherapy [30]. Combination of EBRT with chemotherapy produces a successful therapy to treat OSCC [31]. EBRT can also be used as adjuvant therapy in patients with advanced OSCC after surgery to increase the life expectancy in patients [30].

\section{Drawbacks After Conventional Chemotherapy}

The OSCC cases are increasing in the globe with an alarming rate and the consequence is the increased mortality rate. In addition to recent advancement in the early diagnosis of OSCC, still there is a challenge to provide highly effective therapies [32]. The antcancer drugs generally have numerous drawbacks including narrow therapeutic index, multiple drug resistance (MDR), and severe side effects due to non-specific biodistribution of the drug in the patient's body [32]. The mentioned limitation of the conventional chemotherapy leads to suboptimal dosing, elongation of treatment phase or discontinuation, and poor patient compliance [32]. There are numerous difficulties in the treatment of OSCC, which leads to increased mortality in patients. First of all, it is suggested that most of the cancerous cells arise from a single cell with stem cells property, and the conventional therapy assumes that all somatic cells possess the same malignant potential. An anti-neoplastic drug generally acts on the bulk of tumor cells leaving behind these cancerstem cells, which leads to the recurrence of the disease years after the therapy [33]. Secondly, as the normal cells develop resistance against certain 
stimuli upon repeated stimulation, similarly, most of the cancer cells including the OSCC cells sometimes develop this resistance during the treatment phase, which makes their eradication difficult [34]. Thirdly, early diagnosis of OSCC is very rare and most of the times the disease reaches the advanced stage and spreads to various parts during the therapy, which make it impossible to save the patient's life. Apart from it, unavailability of effective biomarker and non-specific drug targeting normal cells during chemotherapy alongside of metastasis makes it very difficult to treat OSCC.

The OSCC is generally treated with conventional chemotherapy, but such therapies generally have serious blowbacks by their loco-regionally recurrence contributing to high mortality rate [35]. Likewise, another dilemma in the treatment of OSCC is the high incidence of second primary tumors, which affects the survival rate of patients with limited primary tumors [35]. Thus, the postoperative patients who are at higher risk of loco-regional recurrence are good candidates for adjuvant radiation in order to expand their life expectancy [36]. The low survival rate of patients with OSCC is attributed to its poor prognosis and it is reported that the fiveyear survival rate of OSCC varies from $30 \%$ to $40 \%$ [37]. In patients with advanced OSCC and multiple cervical lymph node metastases, the five-year survival rate further decreases to $50 \%$ compared to patients with the absence of lymph node metastasis [37]. It is reported that more than $50 \%$ of patients with OSCC develop local recurrence or metastasis generally within completion of the first two years of treatment [37]. Thus, the overall survival rate decreases in patients with stage 5 cancer.

\section{Cutting Edge of Nanotechnology in Chemotherapy}

Nanoparticles are the class of sub-colloidal particles whose sizes range from 1 to $100 \mathrm{~nm}$. The particles have distinct physicochemical property, attracting researchers to drug delivery purposes. These particles are used in drug delivery, apart from disease diagnosis and imaging. The nanoparticles are widely exploited as a carrier molecule for different therapeutic agents. The drug delivery for the specific site is the method by which the drug gets accumulated at the target and this ability is independent of the route of drug administration [38]. Nanoparticles have the potency to easily cross biological barriers within the human body and thus, it helps to deliver the drug payload at desired site. For effective drug delivery by nanoparticles, four issues are addressed including retaining the drug molecule by the carrier nanoparticles within the human body, escaping the immune system, reaching the target site, and effective release of the drug molecule at the target site [39]. The drug delivery at the nano-scale is studied extensively due to its numerous advantages including its ability to influence the properties such as drug solubility, drug release, bioavailability, immunogenicity; and in addition, overcoming barriers such as opsonization by mononuclear phagocytic system [40]. Targeting of drug molecules with nanoparticles basically includes active and passive targeting, where passive targeting includes forming a drug-carrier complex and active targeting involves specific conjugation to a substrate and receptor [41]. The shape and size of nanoparticles are important parameters that determine their targeting and delivery property to specific site with a controlled transport behavior. The shape and size of the nanoparticles influence the behavior of the target cells and their response for internalization to the cells [42]. Besides, the shape and size also affect the surface to volume ratio of the nanoparticles, which have impact on its pharmacokinetic property [43]. It is reported that the shape and size of nanoparticles affect their dynamicity in the blood vessel towards their walls [44]. In 1990s, Adagen $\AA$, a polyethylene-glycolbased bovine adenosine deaminase, was approved as the first nanotherapeutic agent. More than 40 nanoparticles are approved throughout the globe for clinical use and more than 200 nanoparticles are under different clinical trial phases [45]. It is recently reported that $20 \%-30 \%$ of the nanoparticles of phase-I pass through phase-II and enter phaseIII, and approximately $40 \%-50 \%$ of nanoparticles are marketed [45]. The lipid-based nanocarriers such as liposomes and micelles regarded as the first generation nanoparticles are approved by the FDA [46].

\section{Nanoparticles and Their Application in OSCC Diagnosis and Therapy}

Nanoparticles can attract the attention of the researchers worldwide for their application in the drug delivery systems. These nanoparticles act as a 
novel tool to deliver the drug to the specific site in a controlled manner. Different types of nanoparticles are evolved in the due course of time to treat many diseases including cancer. The nanoparticles are employed to diagnose and treat OSCC. They are employed in biosensors for diagnosis used for detection of a signal molecule, which have good accuracy and convert biochemical signals to electrical signals [47]. Oral fluid nanosensor test is also applied for the multiplex detection of salivary biomarkers of OSCC [48]. Optical nanobiosensors are generally fiber optic tools involved in the analysis of cytochrome $\mathrm{C}$ in apoptosis [49]. With the application of nanotechnology in the field of cancer diagnosis, various types of nanoparticles are applied as contrast agents in specific magnetic resonance imaging (MRI). These nano-contrast agents have the capability to recognize a specific surface marker and they also enhance blood circulation half-life; thus, aid in better MRI contrast properties. It is reported that preconjugated chitosan and magnetic poly (lactide-coglycolide) (PLGA) nanoparticles are used to create MRI contrast in OSCC [50]. Optical coherence tomography (OCT) is a promising tool to diagnose OSCC where gold nanoparticles are used as efficient contrast agent [50]. Photoacoustic imaging is a novel diagnostic technology employed for OSCC where gold nanoparticles act as more attractive contrasting agents due to their capability to conjugate with biomeolecules as compared to other contrast agents such as methylene blue [50]. Raman spectroscopy is employed in the diagnosis of OSCC based on the concept of differences in the scattering of light between normal, premalignant, and malignant lesions. Recently, surface-enhanced Raman (SERS) spectroscopy gold nanoparticles were employed for single OSCC imaging; moreover, they can selectively target intracellular organelles too [50].

Quantum dots are termed as nano-sized semiconducting crystals, which luminesce via quantum confinement effects. It is reported that quantum dots are employed in molecular and cell imaging of OSCC as well as personalized therapy of OSCC [50]. Detection and investigation of many biomarkers including tumor necrosis factoralpha and vascular endothelial growth factor helps in early detection of OSCC. The applications of nanotechnology streamline the process of early detection of biomarkers. It is reported that saliva peptide finger print technique employed nanotechnology for early detection of OSCC [50]. Lymphotropic nanoparticles are considered as novel class of MRI contrast agent that is also reported to have improved diagnostic examination sensitivity of OSCC [51]. Copper (64Cu) liposomes are reported to act as a radiotracer to augment visualization and detection of OSCC [52]. Few other diagnostic tools employed for OSCC detection include nanoscale cantilevers, which are elastic beams used to attach to cancer-linked molecules, cantilever array sensors, nanopores, nanotubes, and multiplex modality [53]. Polymeric nanoparticles recently gained considerable attention for their applications in drug delivery. As they are highly biocompatible and efficacious, and show great bioavailability of the entrapped drug molecule and apart from that have simple elaboration of design and broad structural variety, they are highly exploited in drug delivery. Due to their sizes in the nanometer range, they are able to penetrate and permeate across the cell membrane, blood capillaries, and biological barriers; moreover, their sustained drug release nature until they reach the target size makes them much more suitable for drug delivery [54]. Among different polymeric nanoparticles, poly (lactic-coglycolic acid) (PLGA) is an emerging and effective biocompatible polymer used for the preparation of nanoparticles. They are employed in drug targeting, imaging, diagnosis, and therapy. And the foremost advantage of PLGA is their complete degradation in the aqueous medium [55]. Apart from PLGA, polyethylene glycol (PEG) with distinct characteristics and safety profile is also approved by FDA for use in human [56]. Polymeric nanoparticles based on poly (ethylene glycol)-poly (glutamic acid) block copolymers are used for effective delivery of cisplatin in OSCC [57]. It is reported that PEGylated titanium dioxides are loaded to inhibit proliferation, angiogenesis, and metastasis in OSCC cases [58]. It is also reported that $\mathrm{pH}$-sensitive poly (2-[methacryloyloxy]ethyl phosphorylcholine) (PDPA)-poly [2-diisopropylamino]ethyl methacrylate) (PMPC) polymersomes successfully entrap and deliver anticancer drugs against OSCC [59].

Chitosan is a natural polymer and, due to its biodegradability and biocompatibility, is employed 
in the formulation of microspheres and nanoparticles [60]. Chitosan nanoparticles prepared in combination with arginyl-glycyl-aspartic acid (RGD) displayed high cellular intake via integrin and receptormediated endocytosis led to enhanced cytotoxicity to target cells [61]. Natural compounds such as ellagic acid encapsulated within chitosan nanoparticles are evaluated against OSCC [62]. It is reported that modified chitosan nanoparticles entrapping 5 -aminolevulinic acid are effectively taken by the OSCC cells via folate receptor-mediated endocytosis [63]. Polymeric micelles have unique characteristics including the ability to deliver drugs to the cancer cells. Micelles are mostly composed of diblock or triblock copolymers; graft copolymers with both the hydrophilic and hydrophobic nature are studied for the drug delivery against OSCC. They generally have some unique properties including low molecular weight, more stability of the drug, lower critical micelle concentration, more drug encapsulation, and most importantly, high extent of drug accumulation at the target site, which make them a more suitable drug carrier [64]. Dendrimers are a newer class of polymeric drug delivery systems basically used for chemotherapy drugs and theranostics against OSCC. They are actually synthetic polymers made up with monomer units and are also regarded as the polymers of the 21 st century [65]. Nanoconjugates made up with drug and polymers are watersoluble and even biocompatible and are linked to each other through biological linking and even target molecule. Polybutyl cyanoacrylate-based nanoparticles are extensively studied among all the biomaterial used for drug delivery due to their high biodegradability and non-toxicity to human body. They are mostly used in the clinical trials conducted across the world [66]. Albumin is a class of protein generally obtained from oval albumin, bovine serum albumin, and human serum albumin. Due to its non-toxic, biocompatible, biodegradable, greater water solubility, and non-immunogenic nature, it is employed to prepare nanoparticles in cancer therapy, specifically for drugs such as Paclitaxel [67]. In 1991, the first lipid-based nanocarriers were introduced as an alternative to emulsion, microparticles and polymeric nanoparticles. They are mostly formulated by dispersing the lipids in the aqueous surfactant solution in water or by dispersion of lipids in water. The lipid- based nanoparticles are highly biocompatible and biodegradable by nature [68]. In 1995, liposomes were first approved as an advanced drug delivery system. Improved pharmacokinetic nature, low toxicity, and enhanced targeting effect were among the few advantages of liposomes as a drug carrier for anticancer drug delivery [69]. Another class of lipidbased drug delivery systems includes cubosome and transferosome, characterized with high surface area and low viscosity [70]. Solid lipid nanoparticles (SLN) were among the ones employed to carry hydrophobic drugs. These particles were generally composed of solid lipids, waxes, glycerides, and fatty acids and they are fixed by a surfactant. The ability to optimize the drug payload, sustained release profile, low toxicity, drug inertness and target specificity make them efficient drug carriers [71]. Nanostructured lipid carriers (NLC) are another class of lipid-based carriers composed of a solid and a liquid lipid. High drug payload, sustained release nature, and biocompatibility are some of their advantages [72]. SLN and NLC also act as a potential delivery tool for anticancer drugs against OSCC [73, 74].

Recently, inorganic nanoparticles attractedattention due to their distinct physicochemical properties that are better than those of polymeric and lipid nanoparticles. Due to their capability to achieve physical and chemical surface modification, gold, mesoporous silica, nanoprecipitate, nanoemulsion, and iron oxide nanoparticles are highly applicable in theranostics, diagnosis, drug delivery, and bioimaging $[75,76]$. Nanoprecipitation techniques generating polycaprolactone nanoparticles are explored for curcumin delivery in the oral cavity [77]. Nanoemulsions loaded with genistein were effective against OSCC cell lines [78]. Anti-epithelial growth factor receptor antibody conjugated to gold nanoparticles were fabricated and investigated against OSCC cell lines [79]. It is reported that mesoporous silica nanoparticles act as effective carrier molecules for anticancer drugs in OSCC therapy [80]. Apart from the abovementioned nanoparticles, continuous efforts and investigations are always under progress to explore newer types of nanoparticles. Nanocapsules, nanogels, and silk fibrion are among a few of newly investigated nanoparticles studied in chemotherapy $[81,82]$. 


\section{CONCLUSION}

OSCC is recognized as one of common types of cancer with multi-factorial determinants for its development. The poor diagnosis of OSCC and failure of conventional therapy results in loss of millions of human lives throughout the globe every year. The present scenario of treatment for OSCC mainly has undesired side effects as well as recurrence of OSCC after the completion of treatment. Nanotechnology is a novel tool in terms of drug-delivery and disease diagnosis. The nanoparticles can be effectively used in anticancer drug delivery, basically due to their inherent physicochemical properties. The inherent property of nanoparticles assures successful delivery of the anti-neoplastic drugs to the tumor cells; they apparently open many scopes to deliver diverse types of anti-neoplastic drugs. The nanotechnology and nanoparticles can be effectively employed to treat OSCC by combating the drawbacks associated with the conventional chemotherapy.

\section{ACKNOWLEDGEMENTS}

Not applicable.

\section{CONFLICT OF INTEREST}

The authors have no conflicts of interest to declare.

\section{ETHICS APPROVAL}

Not applicable.

\section{REFERENCES}

1. Stratton MR, Campbell PJ, Futreal PA. The cancer genome. Nature. 2009;458(7239):719-24. DOI: 10.1038/nature07943 PMID: 19360079.

2. Aktipis CA, Nesse RM. Evolutionary foundations for cancer biology. Evol Appl. 2013;6(1):144-59. DOI: 10.1111/ eva.12034 PMID: 23396885.

3. Strassmann JE, Queller DC. The social organism: congresses, parties, and committees. Evolution. 2010;64(3):60516. DOI: $10.1111 / \mathrm{j} .1558-5646.2009 .00929 . \mathrm{x}$ PMID: 20100213 .

4. Mohme M, Riethdorf S, Pantel K. Circulating and disseminated tumour cells - mechanisms of immune surveillance and escape. Nat Rev Clin Oncol. 2017;14(3):155-67. DOI: 10.1038/nrclinonc.2016.144 PMID: 27644321.

5. Ferlay J, Colombet M, Soerjomataram I, Mathers C, Parkin DM, Pineros M, et al. Estimating the global cancer incidence and mortality in 2018: GLOBOCAN sources and methods. Int J Cancer. 2019;144(8):1941-53. DOI: 10.1002/ijc.31937 PMID: 30350310.

6. Lingen MW, Kalmar JR, Karrison T, Speight PM. Critical evaluation of diagnostic aids for the detection of oral cancer. Oral Oncol. 2008;44(1):10-22. DOI: 10.1016/j.oraloncology.2007.06.011 PMID: 17825602.

7. Siegel RL, Miller KD, Fedewa SA, Ahnen DJ, Meester RGS, Barzi A, et al. Colorectal cancer statistics, 2017. CA Cancer J Clin. 2017;67(3):177-93. DOI: 10.3322/ caac.21395 PMID: 28248415.

8. Barnes L, Eveson JW, Sidransky D, Reichart P. Pathology and Genetics of Head and Neck Tumours: IARC Press; 2005.

9. Murphy BA, Gilbert J, Cmelak A, Ridner SH. Symptom control issues and supportive care of patients with head and neck cancers. Clin Adv Hematol Oncol. 2007;5(10):80722. PMID: 17998898.

10. McGurk M, Chan C, Jones J, O'Regan E, Sherriff M. Delay in diagnosis and its effect on outcome in head and neck cancer. Br J Oral Maxillofac Surg. 2005;43(4):281-4. DOI: 10.1016/j.bjoms.2004.01.016 PMID: 15993279.

11. Liu X, Kolokythas A, Wang J, Huang H, Zhou X. Gene Expression Signatures of Lymph Node Metastasis in Oral Cancer: Molecular Characteristics and Clinical Significances. Curr Cancer Ther Rev. 2010;6(4):294-307. DOI: 10.2174/157339410793358066 PMID: 21709736.

12. van Zyl AW, Marnewick JC. Aetiology of oral cancer. SADJ. 2012;67(10):554-6. PMID: 23957094.

13. IARC Working Group on the Evaluation of Carcinogenic Risk to Humans. Tobacco Smoke and Involuntary Smoking. IARC Monographs on the Evaluation of Carcinogenic Risks to Humans. Lyon (FR): International Agency for Research on Cancer; 2004. 1-1438 p.

14. IARC Working Group on the Evaluation of Carcinogenic Risk to Humans. Betel-quid and Areca-nut Chewing and Some Areca-nut-derived Nitrosamines. IARC Monographs on the Evaluation of Carcinogenic Risks to Humans. Lyon (FR): International Agency for Research on Cancer; 2004. 1-349 p.

15. IARC Working Group on the Evaluation of Carcinogenic Risk to Humans. Alcohol Consumption and Ethyl Carbamate. IARC Monographs on the Evaluation of Carcinogenic Risks to Humans. Lyon (FR): International Agency for Research on Cancer; 2010. 1-1440 p.

16. Mehanna H, Beech T, Nicholson T, El-Hariry I, McConkey C, Paleri V, et al. Prevalence of human papillomavirus in oropharyngeal and nonoropharyngeal head and neck cancer--systematic review and meta-analysis of trends by time and region. Head Neck. 2013;35(5):747-55. DOI: 10.1002/ hed.22015 PMID: 22267298.

17. Hashibe M, Brennan P, Benhamou S, Castellsague X, Chen C, Curado MP, et al. Alcohol drinking in never users of tobacco, cigarette smoking in never drinkers, and the risk of head and neck cancer: pooled analysis in the International Head and Neck Cancer Epidemiology Consortium. J Natl Cancer Inst. 2007;99(10):777-89. DOI: 10.1093/jnci/ djk179 PMID: 17505073.

18. Rodriguez T, Altieri A, Chatenoud L, Gallus S, Bosetti C, Negri E, et al. Risk factors for oral and pharyngeal cancer in young adults. Oral Oncol. 2004;40(2):207-13. DOI: 10.1016/j.oraloncology.2003.08.014 PMID: 14693246.

19. Schutze M, Boeing H, Pischon T, Rehm J, Kehoe T, Gmel 
$\mathrm{G}$, et al. Alcohol attributable burden of incidence of cancer in eight European countries based on results from prospective cohort study. BMJ. 2011;342:d1584. DOI: 10.1136/ bmj.d1584 PMID: 21474525.

20. Sullivan RJ, Hagen EH. Psychotropic substance-seeking: evolutionary pathology or adaptation? Addiction. 2002;97(4):389-400. DOI: 10.1046/j.13600443.2002.00024.x PMID: 11964056.

21. Gupta PC, Warnakulasuriya S. Global epidemiology of areca nut usage. Addict Biol. 2002;7(1):77-83. DOI: 10.1080/13556210020091437 PMID: 11900626.

22. Hoffmann D, Hoffmann I. Smoking and Tobacco Control, Monograph 9: Cigars Health Effects and Trends. Washington, D.C.: National Cancer Institute; 1998.

23. Petersen PE, Bourgeois D, Ogawa H, Estupinan-Day S, Ndiaye C. The global burden of oral diseases and risks to oral health. Bull World Health Organ. 2005;83(9):661-9. DOI: /s0042-96862005000900011 PMID: 16211157.

24. Warnakulasuriya S. Global epidemiology of oral and oropharyngeal cancer. Oral Oncol. 2009;45(4-5):309-16. DOI: 10.1016/j.oraloncology.2008.06.002 PMID: 18804401.

25. Boyle P, Ferlay J. Cancer incidence and mortality in Europe, 2004. Ann Oncol. 2005;16(3):481-8. DOI: 10.1093/ annonc/mdi098 PMID: 15718248.

26. Carneiro-Neto JN, de-Menezes JD, Moura LB, Massucato EM, de-Andrade CR. Protocols for management of oral complications of chemotherapy and/or radiotherapy for oral cancer: Systematic review and meta-analysis current. Med Oral Patol Oral Cir Bucal. 2017;22(1):e15-e23. DOI: 10.4317/medoral.21314 PMID: 27918734.

27. Shah JP, Gil Z. Current concepts in management of oral cancer--surgery. Oral Oncol. 2009;45(4-5):394-401. DOI: 10.1016/j.oraloncology.2008.05.017 PMID: 18674952.

28. Moding EJ, Kastan MB, Kirsch DG. Strategies for optimizing the response of cancer and normal tissues to radiation. Nat Rev Drug Discov. 2013;12(7):526-42. DOI: 10.1038/ nrd4003 PMID: 23812271.

29. Huang SH, O’Sullivan B. Oral cancer: Current role of radiotherapy and chemotherapy. Med Oral Patol Oral Cir Bucal. 2013;18(2):e233-40. DOI: 10.4317/medoral.18772 PMID: 23385513.

30. Cabrera-Rodríguez JJ. The role of radiotherapy in the treatment of oral cavity cancer. Plast Aesthet Res. 2016;3:15866. DOI: https://doi.org/10.20517/2347-9264.2016.13.

31. Blanchard P, Baujat B, Holostenco V, Bourredjem A, Baey $\mathrm{C}$, Bourhis J, et al. Meta-analysis of chemotherapy in head and neck cancer (MACH-NC): a comprehensive analysis by tumour site. Radiother Oncol. 2011;100(1):33-40. DOI: 10.1016/j.radonc.2011.05.036 PMID: 21684027.

32. Glasgow MD, Chougule MB. Recent Developments in Active Tumor Targeted Multifunctional Nanoparticles for Combination Chemotherapy in Cancer Treatment and Imaging. J Biomed Nanotechnol. 2015;11(11):1859-98. DOI: 10.1166/jbn.2015.2145 PMID: 26554150.

33. Copland M, Jorgensen HG, Holyoake TL. Evolving molecular therapy for chronic myeloid leukaemia--are we on target? Hematology. 2005;10(5):349-59. DOI: $\underline{10.1080 / 10245330500234195}$ PMID: 16203604.

34. Raaijmakers MH, de Grouw EP, Heuver LH, van der Reijden BA, Jansen JH, Scheper RJ, et al. Breast cancer resistance protein in drug resistance of primitive CD34+38- cells in acute myeloid leukemia. Clin Cancer Res. 2005;11(6):2436-44. DOI: 10.1158/1078-0432.CCR04-0212 PMID: 15788695.

35. Liu CH, Chen HJ, Wang PC, Chen HS, Chang YL. Patterns of recurrence and second primary tumors in oral squamous cell carcinoma treated with surgery alone. Kaohsiung J Med Sci. 2013;29(10):554-9. DOI: 10.1016/j.kjms.2013.03.001 PMID: 24099110.

36. Hinerman RW, Mendenhall WM, Morris CG, Amdur RJ, Werning JW, Villaret DB. Postoperative irradiation for squamous cell carcinoma of the oral cavity: 35-year experience. Head Neck. 2004;26(11):984-94. DOI: 10.1002/ hed.20091 PMID: 15459927.

37. Gupta S, Kushwaha VS, Verma S, Khan H, Bhatt ML, Husain N, et al. Understanding molecular markers in recurrent oral squamous cell carcinoma treated with chemoradiation. Heliyon. 2016;2(12):e00206. DOI: 10.1016/j. heliyon.2016.e00206 PMID: 27981249.

38. Torchilin VP. Drug targeting. Eur J Pharm Sci. 2000;11:S81-S91. DOI: https://doi.org/10.1016/S09280987(00)00166-4 PMID: 11033430.

39. Mills JK, Needham D. Targeted drug delivery. Expert Opin Ther Pat. 1999;9(11):1499-513. DOI: 10.1517/13543776.9.11.1499.

40. Blanco E, Shen H, Ferrari M. Principles of nanoparticle design for overcoming biological barriers to drug delivery. Nat Biotechnol. 2015;33(9):941-51. DOI: 10.1038/ nbt.3330 PMID: 26348965.

41. Bae YH, Park K. Targeted drug delivery to tumors: myths, reality and possibility. J Control Release. 2011;153(3):198205. DOI: 10.1016/j.jconrel.2011.06.001 PMID: 21663778.

42. Lamprecht A, Schafer U, Lehr CM. Size-dependent bioadhesion of micro- and nanoparticulate carriers to the inflamed colonic mucosa. Pharm Res. 2001;18(6):788-93. DOI: $10.1023 / \mathrm{a}: 1011032328064$ PMID: 11474782.

43. Panyam J, Dali MM, Sahoo SK, Ma W, Chakravarthi SS, Amidon GL, et al. Polymer degradation and in vitro release of a model protein from poly(d,1-lactide-coglycolide) nano- and microparticles. J Control Release. 2003;92(1):173-87. DOI: https://doi.org/10.1016/S01683659(03)00328-6 PMID: 14499195.

44. Decuzzi P, Lee S, Bhushan B, Ferrari M. A theoretical model for the margination of particles within blood vessels. Ann Biomed Eng. 2005;33(2):179-90. DOI: 10.1007/ s10439-005-8976-5 PMID: 15771271.

45. Schutz CA, Juillerat-Jeanneret L, Mueller H, Lynch I, Riediker M, NanoImpactNet C. Therapeutic nanoparticles in clinics and under clinical evaluation. Nanomedicine (Lond). 2013;8(3):449-67. DOI: 10.2217/nnm.13.8 PMID: 23477336.

46. Shi X, Sun K, Baker JR. Spontaneous Formation of Functionalized Dendrimer-Stabilized Gold Nanoparticles. J Phys Chem C Nanomater Interfaces. 2009;112(22):82518. DOI: $10.1021 / \mathrm{jp} 801293 \mathrm{a}$ PMID: 19727334. 
47. Saravana KR, Vijayalakshmi R. Nanotechnology in dentistry. Indian J Dent Res. 2006;17(2):62-5. DOI: 10.4103/0970-9290.29890 PMID: 17051869.

48. Gau V, Wong D. Oral fluid nanosensor test (OFNASET) with advanced electrochemical-based molecular analysis platform. Ann N Y Acad Sci. 2007;1098:401-10. DOI: 10.1196/annals.1384.005 PMID: 17435145.

49. Song JM, Kasili PM, Griffin GD, Vo-Dinh T. Detection of cytochrome $\mathrm{C}$ in a single cell using an optical nanobiosensor. Anal Chem. 2004;76(9):2591-4. DOI: 10.1021/ ac0352878 PMID: 15117202.

50. Chen XJ, Zhang XQ, Liu Q, Zhang J, Zhou G. Nanotechnology: a promising method for oral cancer detection and diagnosis. J Nanobiotechnology. 2018;16(1):52. DOI: 10.1186/s12951-018-0378-6 PMID: 29890977.

51. De Felice F, Cavallini C, Barlattani A, Tombolini M, Brugnoletti O, Tombolini V, et al. Nanotechnology in Oral Cavity Carcinoma: Recent Trends and Treatment Opportunities. Nanomaterials (Basel). 2019;9(11). DOI: 10.3390/ nano9111546 PMID: 31683582.

52. Mahakian LM, Farwell DG, Zhang H, Seo JW, Poirier B, Tinling SP, et al. Comparison of PET imaging with 64Cu-liposomes and 18F-FDG in the 7,12-dimethylbenz[a] anthracene (DMBA)-induced hamster buccal pouch model of oral dysplasia and squamous cell carcinoma. Mol Imaging Biol. 2014;16(2):284-92. DOI: 10.1007/s11307-0130676-1 PMID: 24019092.

53. Poonia M, Ramalingam K, Goyal S, Sidhu SK. Nanotechnology in oral cancer: A comprehensive review. J Oral Maxillofac Pathol. 2017;21(3):407-14. DOI: 10.4103/jomfp.JOMFP $29 \quad 17$ PMID: 29391716.

54. El-Say KM, El-Sawy HS. Polymeric nanoparticles: Promising platform for drug delivery. Int J Pharm. 2017;528(12):675-91. DOI: 10.1016/j.ijpharm.2017.06.052 PMID: 28629982.

55. Peres C, Matos AI, Conniot J, Sainz V, Zupancic E, Silva JM, et al. Poly(lactic acid)-based particulate systems are promising tools for immune modulation. Acta Biomater. 2017;48:41-57. DOI: 10.1016/j.actbio.2016.11.012 PMID: 27826003.

56. D’Souza A A, Shegokar R. Polyethylene glycol (PEG): a versatile polymer for pharmaceutical applications. Expert Opin Drug Deliv. 2016;13(9):1257-75. DOI: 10.1080/17425247.2016.1182485 PMID: 27116988.

57. Endo K, Ueno T, Kondo S, Wakisaka N, Murono S, Ito $\mathrm{M}$, et al. Tumor-targeted chemotherapy with the nanopolymer-based drug NC-6004 for oral squamous cell carcinoma. Cancer Sci. 2013;104(3):369-74. DOI: 10.1111/ cas.12079 PMID: 23216802.

58. Lucky SS, Idris NM, Huang K, Kim J, Li Z, Thong PS, et al. In vivo Biocompatibility, Biodistribution and Therapeutic Efficiency of Titania Coated Upconversion Nanoparticles for Photodynamic Therapy of Solid Oral Cancers. Theranostics. 2016;6(11):1844-65. DOI: 10.7150/thno.15088 PMID: 27570555 .

59. Colley HE, Hearnden V, Avila-Olias M, Cecchin D, Canton I, Madsen J, et al. Polymersome-mediated delivery of combination anticancer therapy to head and neck cancer cells: 2D and 3D in vitro evaluation. Mol Pharm. 2014;11(4):1176-88. DOI: 10.1021/mp400610b PMID: 24533501.

60. Quinones JP, Peniche H, Peniche C. Chitosan Based Self-Assembled Nanoparticles in Drug Delivery. Polymers (Basel). 2018;10(3). DOI: 10.3390/polym10030235 PMID: 30966270.

61. Babu A, Amreddy N, Muralidharan R, Pathuri G, Gali H, Chen A, et al. Chemodrug delivery using integrin-targeted PLGA-Chitosan nanoparticle for lung cancer therapy. Sci Rep. 2017;7(1):14674. DOI: 10.1038/s41598-017-15012-5 PMID: 29116098.

62. Arulmozhi V, Pandian K, Mirunalini S. Ellagic acid encapsulated chitosan nanoparticles for drug delivery system in human oral cancer cell line (KB). Colloids Surf B Biointerfaces. 2013;110:313-20. DOI: 10.1016/j.colsurfb.2013.03.039 PMID: 23732810.

63. Nguyen S, Hiorth M. Advanced drug delivery systems for local treatment of the oral cavity. Ther Deliv. 2015;6(5):595608. DOI: $10.4155 /$ tde.15.5 PMID: 26001175.

64. Deshmukh AS, Chauhan PN, Noolvi MN, Chaturvedi K, Ganguly K, Shukla SS, et al. Polymeric micelles: Basic research to clinical practice. Int J Pharm. 2017;532(1):24968. DOI: 10.1016/j.ijpharm.2017.09.005 PMID: 28882486.

65. Singh J, Jain K, Mehra NK, Jain NK. Dendrimers in anticancer drug delivery: mechanism of interaction of drug and dendrimers. ArtifCells Nanomed Biotechnol. 2016;44(7):162634. DOI: 10.3109/21691401.2015.1129625 PMID: $\underline{26747336 .}$.

66. Gao S, Xu Y, Asghar S, Chen M, Zou L, Eltayeb S, et al. Polybutylcyanoacrylate nanocarriers as promising targeted drug delivery systems. J Drug Target. 2015;23(6):481-96. DOI: 10.3109/1061186X.2015.1020426 PMID: 25738991.

67. Kudarha RR, Sawant KK. Albumin based versatile multifunctional nanocarriers for cancer therapy: Fabrication, surface modification, multimodal therapeutics and imaging approaches. Mater Sci Eng C Mater Biol Appl. 2017;81:607-26. DOI: 10.1016/j.msec.2017.08.004 PMID: 28888016.

68. Patil AM, Todkar RR, Dipak GS, Mohite S, Magdum C. A review on solid lipid nanoparticle. Res J Pharm Dosage Forms Tech. 2016;8(3):218-20. DOI: 10.5958/09754377.2016.00030.6.

69. Kuang Y, Liu J, Liu Z, Zhuo R. Cholesterol-based anionic long-circulating cisplatin liposomes with reduced renal toxicity. Biomaterials. 2012;33(5):1596-606. DOI: 10.1016/j. biomaterials.2011.10.081 PMID: 22079777.

70. Saber MM, Al-Mahallawi AM, Nassar NN, Stork B, Shouman SA. Targeting colorectal cancer cell metabolism through development of cisplatin and metformin nano-cubosomes. BMC Cancer. 2018;18(1):822. DOI: 10.1186/ s12885-018-4727-5 PMID: 30111296.

71. Fangueiro JF, Gonzalez-Mira E, Martins-Lopes P, Egea MA, Garcia ML, Souto SB, et al. A novel lipid nanocarrier for insulin delivery: production, characterization and toxicity testing. Pharm Dev Technol. 2013;18(3):545-9. DOI: 
10.3109/10837450.2011.591804 PMID: 21711084.

72. Obeidat WM, Schwabe K, Muller RH, Keck CM. Preservation of nanostructured lipid carriers (NLC). Eur J Pharm Biopharm. 2010;76(1):56-67. DOI: 10.1016/j. ejpb.2010.05.001 PMID: 20452422.

73. Liu D, Liu Z, Wang L, Zhang C, Zhang N. Nanostructured lipid carriers as novel carrier for parenteral delivery of docetaxel. Colloids Surf B Biointerfaces. 2011;85(2):2629. DOI: 10.1016/j.colsurfb.2011.02.038 PMID: 21435845.

74. Bharadwaj R, Sahu BP, Haloi J, Laloo D, Barooah P, Keppen $\mathrm{C}$, et al. Combinatorial therapeutic approach for treatment of oral squamous cell carcinoma. Artif Cells Nanomed Biotechnol. 2019;47(1):572-85. DOI: 10.1080/21691401.2019.1573176 PMID: 30831033.

75. Yang C, Bromma K, Sung W, Schuemann J, Chithrani D. Determining the Radiation Enhancement Effects of Gold Nanoparticles in Cells in a Combined Treatment with Cisplatin and Radiation at Therapeutic Megavoltage Energies. Cancers (Basel). 2018;10(5). DOI: 10.3390/cancers10050150 PMID: 29786642.

76. Zhou Y, Quan G, Wu Q, Zhang X, Niu B, Wu B, et al. Mesoporous silica nanoparticles for drug and gene delivery. Acta Pharm Sin B. 2018;8(2):165-77. DOI: 10.1016/j. apsb.2018.01.007 PMID: 29719777.

77. Mazzarino L, Loch-Neckel G, Bubniak Ldos S, Mazzucco S, Santos-Silva MC, Borsali R, et al. Curcumin-Loaded Chitosan-Coated Nanoparticles as a New Approach for the Local Treatment of Oral Cavity Cancer. J Nanosci Nano- technol. 2015;15(1):781-91. DOI: 10.1166/jnn.2015.9189 PMID: 26328442

78. Lin HY, Thomas JL, Chen HW, Shen CM, Yang WJ, Lee $\mathrm{MH}$. In vitro suppression of oral squamous cell carcinoma growth by ultrasound-mediated delivery of curcumin microemulsions. Int J Nanomedicine. 2012;7:941-51. DOI: 10.2147/IJN.S28510 PMID: 22393291.

79. El-Sayed IH, Huang X, El-Sayed MA. Selective laser photo-thermal therapy of epithelial carcinoma using anti-EGFR antibody conjugated gold nanoparticles. Cancer Lett. 2006;239(1):129-35. DOI: 10.1016/j.canlet.2005.07.035 PMID: 16198049 .

80. Wang D, Xu X, Zhang K, Sun B, Wang L, Meng L, et al. Codelivery of doxorubicin and MDR1-siRNA by mesoporous silica nanoparticles-polymerpolyethylenimine to improve oral squamous carcinoma treatment. Int J Nanomedicine. 2018;13:187-98. DOI: 10.2147/IJN.S150610 PMID: 29343957.

81. Gao X, Yang H, Wu M, Shi K, Zhou C, Peng J, et al. Targeting Delivery of Lidocaine and Cisplatin by Nanogel Enhances Chemotherapy and Alleviates Metastasis. ACS Appl Mater Interfaces. 2018;10(30):25228-40. DOI: 10.1021/acsami.8b09376 PMID: 29979563.

82. Qu J, Liu Y, Yu Y, Li J, Luo J, Li M. Silk fibroin nanoparticles prepared by electrospray as controlled release carriers of cisplatin. Mater Sci Eng C Mater Biol Appl. 2014;44:166-74. DOI: 10.1016/j.msec.2014.08.034 PMID: $\underline{25280693 .}$. 